\title{
INDIVIDUAL AND SOCIAL RISK DURING TRANSPORTATION OF DANGEROUS SUBSTANCES
}

This article aims to present new views on risk assessment, primarily individual and social risks during transportation of dangerous substance. Included are modelling procedures and methods applied to calculate dangerous substance exposure to the biodiversity of the environment, solved in project BIOTRA. New procedures and methods applied for calculation of environmental exposure by dangerous substance outflows in road transport are also presented.

\section{Introduction}

The Technical University of Liberec began working on the project "BIOTRA" in 2008. This project is part of the National research program in the Czech Republic. Experts from other institutions cooperate on project solving tasks. The "BIOTRA" project is aimed at producing methodology for ecological risk evaluations associated with dangerous goods transport with special focus on environment and its biotic elements. The main result of the project "BIOTRA" should be the designing of decision making software tools for the ecological transportation alternatives selection, which will also allow determining recommended routes for dangerous goods in road transport.

\section{Threat evaluation regarding road transport}

The investigation of threats regarding road transport is currently one of the central tasks. There are thousands of people dying on the roads every year. The EU has defined in the White book the tasks for membership countries to lower numbers of mortal accidents to the half. According to a statistical annual report, the results of mortality on Slovak roads as followed:

The development of mortality on Slovak roads

Table 1 in $2000-2009$

\begin{tabular}{|l|c|c|c|c|}
\hline \multicolumn{1}{|c|}{ Year } & 2000 & 2003 & 2006 & 2009 \\
\hline Fatalities & 628 & 645 & 519 & 386 \\
\hline Percentage according to plan & $100 \%$ & $102.7 \%$ & $82.6 \%$ & $61.4 \%$ \\
\hline
\end{tabular}

Source: [9]

\footnotetext{
* Zdenek Dvorak ${ }^{1}$, Pavel Fuchs ${ }^{2}$, Jan Novak ${ }^{2}$, Radovan Sousek ${ }^{3}$

${ }^{1}$ University of Zilina, Slovakia, Email: Zdenek.Dvorak@fsi.uniza.sk

${ }^{2}$ Technical University of Liberec, Czech Republic

${ }^{3}$ University of Pardubice, Czech Republic
}

Investigation of accident causes is based on statistical data. The methods are focused on the identification of particular elements in the transport system. Therefore, we evaluated each of following parts separately: Road infrastructure, transport means, transport technology, specific management and information systems in road transport. The findings show that the most crucial deficiency was in the quality and capacity of road infrastructure.

\section{Potential escape of dangerous substances}

The significant threat for transport, human health and the environment is the transport of dangerous substances. There are certain characteristics of such accidents:

- Escape time is limited by the vessel discharge; the worst case is assumed to be the instantaneous outflow of whole tank volume to the environment,

- Substance quantity is limited by transport vessel capacity (road tank, tank car, etc.),

- Outflow location is not known in advance, it may be situated wherever on the transport route.

The bandwidth exposure assessment is simpler if a risk map compilation in the surrounding of the single outflow source or of whole transport route is available. The problem is simplified to risk assessment in the profile perpendicular to the route. In a general case all present conditions are not known, especially atmospheric stability and wind speed. It is necessary to calculate the probability of standard meteorological conditions and these probabilities to an average according to their representation in locality [1], [7]. 


\subsection{Explosions}

Explosion - probability of the leaking of unconfined gas cloud into the atmosphere is considerably smaller than the probability of fire. The basic presumption for explosion is that the concentration of gas must exceed the lower explosion limit.

The explosion sets in a shock wave. The negative effect is overpressure on the shock front expressed in pressure units $(\mathrm{kPa}$ or bar). Duration of action is by its effects mostly neglected during the evaluation.

The recommended methods of effect calculation are based on trinitrotoluene equivalents. The results from the geometric similarities are that the parameters of shock waves for a given explosive system are identical to the equal value of reduced distance :

$$
Z=\frac{R}{W^{1 / 3}}
$$

Where

$Z=$ reduced distance $[\mathrm{m}]$,

$R=$ distance from the explosion epicentre $[\mathrm{m}]$,

$W=$ explosion system weight $[\mathrm{kg}]$, formulated by an equivalent TNT.

From the calculated reduced distance it is possible to figure out the overpressure $(\mathrm{Pa})$ on a shock front. This can be founded on dependence due to the reduction of distance from an explosion epicentre. Various formulations of this relation can be found in many different sources. An example of one of many formulas for calculating overpressure is presented below:

$$
\Delta p=\left(\frac{93,2}{Z}+\frac{383}{Z^{2}}+\frac{1275}{Z^{3}}\right)
$$

Where $\Delta p$ is overpressure $[\mathrm{kPa}]$.

Probit function for human death has a formula:

$$
Y=-16.7+2.03 \cdot \ln (\Delta p)
$$

This relation can be used to find probit parameters, e.g. for eardrums break, building destruction or window break. This helps to define different types of environment elements damage.

\subsection{Fire}

In comparison to toxicity, fire takes place in a relatively small area. It is given by the fact that for the gas ignition there are necessary concentrations between the upper and lower ignition limit. The lower ignition limit is mostly higher than the concentration of dangerous gases [2].

Heat radiation is one of the main consequences which affect the surrounding area of an accident.

Energy released by combustion is given by the formula:

$$
E=Q \cdot \Delta H_{c} \cdot \eta
$$

Where

$E=$ released energy $[\mathrm{kJ}]$,

$Q=$ combustible quantity $[\mathrm{kg}]$

$\Delta H_{c}=$ gas heating power $[\mathrm{kJ} / \mathrm{kg}]$,

$\eta=$ radiation ratio of total released energy [-].

In the case of instantaneous outflow the effect depends on the ignition time because the cloud location, geometry and the gas quantity change over time.

For the effect evaluation it is possible to use probit functions. In the case of fire we can use Eisenberg's formula for human death probability:

$$
Y=-14.9+2.56 \cdot \ln V
$$

The parameters $k_{1}, k_{2}$ in the general probit function are not dependent on any particular substance whose characteristics are calculated in regards to the amount of energy released.

Heat exposure dose $V$ depends on thermal radiation. Thermal radiation evoked by point source in distance $r$ is given by the following formula:

$$
I=\frac{E}{4 \pi \cdot r^{2} \cdot t}
$$

Where

$I=$ thermal radiation intensity $[\mathrm{kJ}]$,

$t=$ duration of action $[\mathrm{s}]$,

$r=$ distance from source $[\mathrm{m}]$.

This formula can be used for the case of instantaneous outflow where the cloud has a semi-ellipsoid form.

From the radiation intensity level and exposure duration it is possible to assess the received thermal radiation dose $V$ :

$$
V=I^{4 / 3} \cdot t
$$

Where

$I \quad=$ heat flux $\left[\mathrm{J} /\left(\mathrm{m}^{2} \cdot \mathrm{s}\right)\right]$,

$t=$ exposure time $[\mathrm{s}]$,

$V=$ received dose (tdu - thermal dose unit, $\left.1 t d u=1\left(\mathrm{~kW} / \mathrm{m}^{2}\right)^{4 / 3} \cdot \mathrm{s}\right)$.

Human death probability can be determined using a probit function that results in thermal dosage. According to the thermal dosage, the probability of different types (burn of 1. and 2. instance) of injury can also be determined. In a similar manner can it be specified for fire consequences for fauna. For stationary objects i.e. buildings, constructions, woods, and plants the probable consequences can be determined on the basis of heat flux whose specific value evokes wood ignition. 


\subsection{Gas dispersion in the atmosphere}

The Pasquill - Gifford model "PUFF" is designed for dispersion modelling in instantaneous outflow conditions. It is a dispersion model with normal (Gauss) distribution of concentrations and Lagrange approach. Focus is placed on monitoring gas element movement in a particular wind field. The gas cloud spread in relation to the wind direction. At first the cloud expands and the gas concentration sinks. Later the cloud volume decreases because more and more gas disperses in insignificant concentrations outside the cloud [2].

Gas dispersion is affected by wind speed and atmospheric stability. The atmospheric stability relates to a vertical temperature drop. It generally holds that when the atmospheric stability grows then escaped substances travel longer distances until their concentration sinks. On that ground the higher stability classes are regarded as "bad" weather conditions for dangerous substance dispersion.

\subsection{Bandwidth for liquids}

Defining bandwidth assessment of negative effects for liquids is more difficult than defining bandwidth for gases. There are more scenarios of liquid spread and also a wider spectrum of potentially threatened environmental elements. On the other hand, the bandwidth is considerably smaller.

The diagram of risk evaluation by dangerous liquid outflow is shown in Fig. 1. After the liquid flows onto the earth's surface, three different processes accompanied with different intensities begin: Flushing, imbitition and vaporization. These processes are necessary to simulate simultaneously, because imbitition and vaporization depend on the surface of a created pool. Whereas the flushing is affected by reduction of liquid, which was absorbed or evaporated. The situation is schematically shown in Fig. 1.

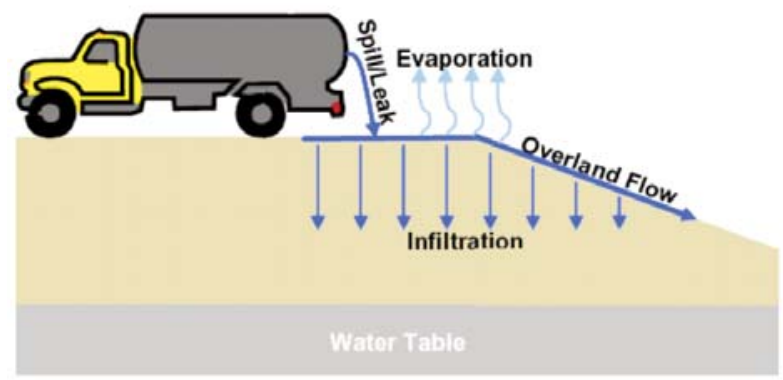

Fig. 1 Diagram of processes by liquid outflow to the terrain

Flushing depends especially on liquid parameters, grade and surface quality, which in general is the width and depth of a pool sink when the grade grows. However, the depth depends on the surface types in discussion. The typical depth on a relatively smooth surface (asphalt, concrete) is so minimal that it can be expressed in millimetres. Vegetation greatly increases the effect of contamina- tion. This creates further surface damage which results from remaining toxic liquids.

For reasons given it is clear that the solution includes two basic types of problems:

- physical problems evaluation,

- finding of necessary area characteristics.

\section{Road transport risk assessment}

Standard methods of traffic effect evaluation of the environment and are oriented towards exhaust and noise assessment. It is also possible to analyse case studies of vehicle accidents connected with dangerous substance outflow. The probability of a major accident is low; however the consequences may be large. Presently there are no methods which allow complex road transport risk assessments to focus on dangerous substance potential outflow consequences for the environment.

The risk is possible to quantify and for its acceptability decision making is this quantification even necessary. Quantitative assessment represents exposure. A numeric value or numeric function can be used to describe the relation between probability and consequences of existing hazards. Various hazards may include machines, activities or technologies. These hazards may also include different objects or processes that endanger humans as well as the environment. Generally, the risk is the product of dangerous event probability and its consequences [3], [4], [8].

\subsection{Outflow calculation and display from stationary sources}

The dispersion models result (air-borne spread, fire, explosion, spilling) is time variable concentration field of escaped substance. Generally, they are calculated in several concentration fields for chosen condition combinations. Concentration field and substance danger parameters are inputs for respondent answer models to negative impact. Negative impacts (toxicity, thermal radiation, shock wave, radioactive radiation) are necessary to be quantified. This is possible by exposure dose calculation which represents physical, chemical and biological effects that depend on substance concentration and action time [2].

\subsection{Social risk}

Social risk $=$ Accident probability $\mathrm{x}$ consequences.

Consequence $=$ exposed human death probability $\mathrm{x}$ the number of threatened people.

Unit individual risk $=$ Individual risk for ever-present unprotected person by unit accident probability.

Unit social risk = Individual risk modified according to presence period, protection level and number of threatened people by unit accident probability. 


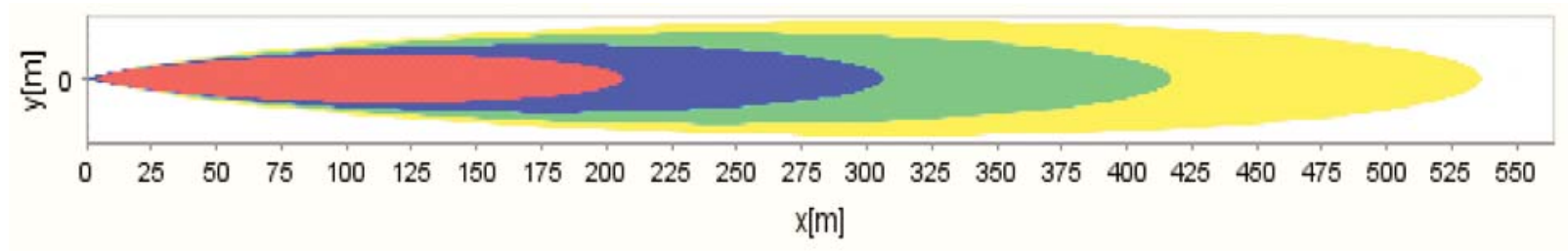

$$
0.95 \backsim 0.5 \backsim 0.1 \backsim 0.01
$$

Fig. 2 Example of human death probability by toxic exposure in dependence on source distance x and y (instantaneous outflow $1000 \mathrm{~kg}$ NH3, atmosphere stability class E, wind speed $1.7 \mathrm{~m} / \mathrm{s}$ )

Death probability is different for group of people according to their protection level (reduced individual risk according to categories).

\section{3 Unit social risk}

$$
S R_{j}=\sum\left(I R_{j} \cdot \sum\left(H_{i} \cdot h_{i}\right)\right)
$$

\section{Whereas}

$I R_{j}$ Represents an individual risk unit in the j-partial area,

$H_{i}=$ number of people i-category in j-area,

$h_{i}=$ people protection level coefficient i-category

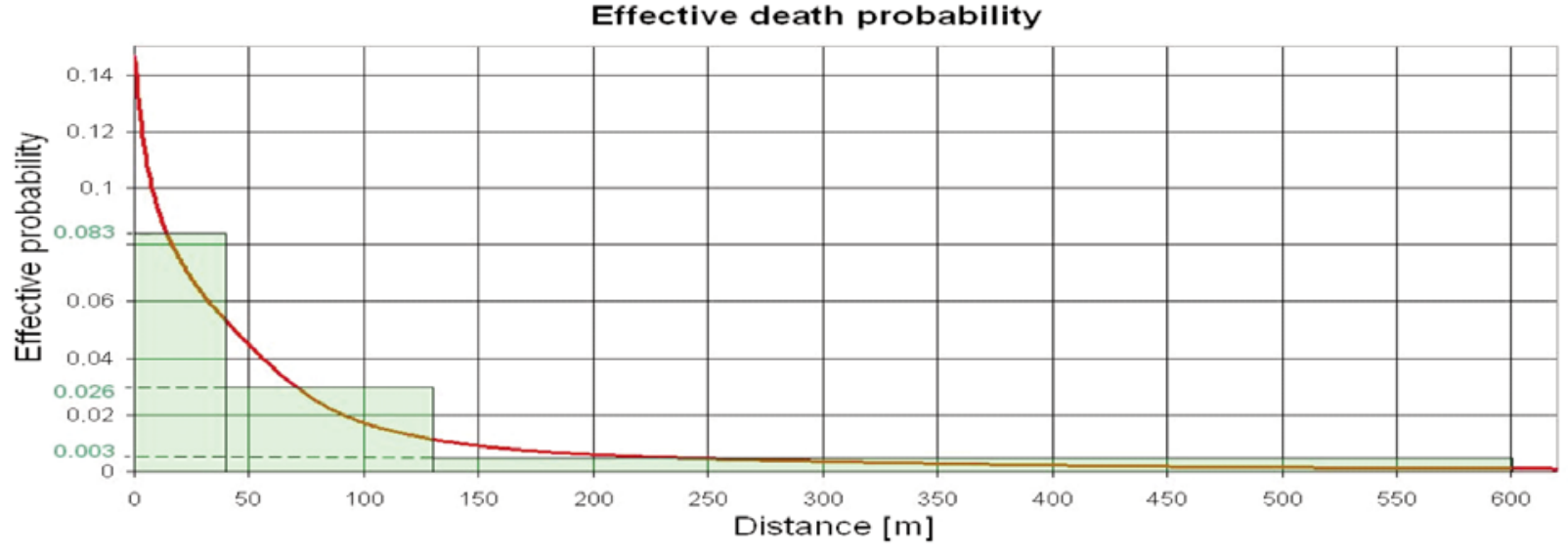

Fig. 3 Effective death probability depending on the distance from the source

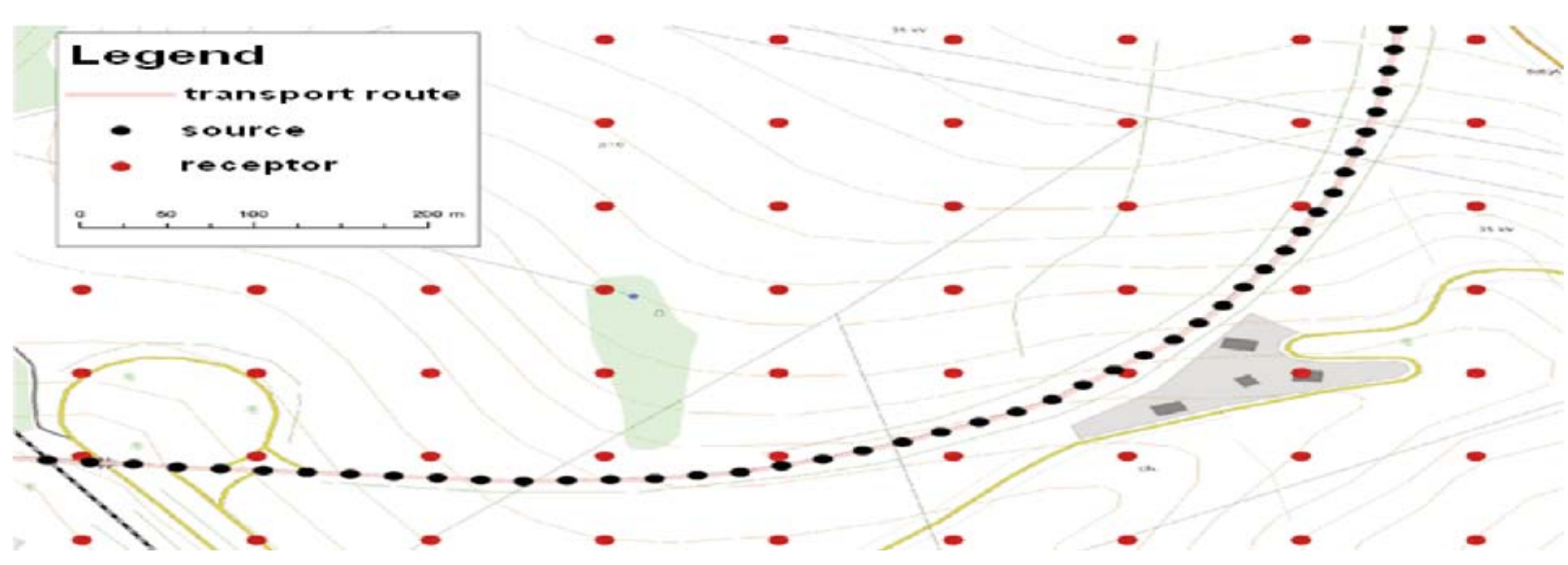

Fig. 4 Schema of segments road distribution and reference point network lay-out. 


\section{Data import to GIS}

Importing data to the GIS software is it useful to define areas with an approximate identical individual risk value. Individual risk decreases from the accident source. If we do not consider wind direction, individual risk is uniformly distributed around a specific source [4].

\subsection{Outflow calculation and display from mobile sources}

Mobile sources move on a defined trajectory. An accident can happen at any point on a trajectory, but only at one point for a particular type of transport. Individual risk at certain point can be expressed as a sum of risks from single road segments. The situation is systematically shown in Fig. 5 [5].
The road is divided into small segments $s$ so that the distance $r$ between reference points $\left[x_{s}, y_{s}\right]$ and each point of a defined segment can be assumed as constant. The cloud width $E(r)$, probability $P(r)$ for a single meteorological situation and average (total) probability $P_{\varphi}(r)$ is constant for a defined segment. The road segmentation should be taken into consideration that the accident probability $P_{h}(s i)$ is constant inside a segment. Then:

$$
P\left(x_{s}, y_{s}\right)=\sum_{i} P_{h}\left(s_{i}\right) \cdot P_{\psi}(r)_{i} \cdot s_{i}
$$

\section{Where}

$P\left(x_{s}, y_{s}\right)=$ total death injury

(lethal dose) probability in a single point $\left[x_{s}, y_{s}\right]$,

$P_{h}\left(s_{i}\right)$ accident probability in a single segment expand to the route unit length $\left[\mathrm{m}^{-1}\right]$

$s_{i} \quad$ length of route a single segment $i[\mathrm{~m}]$,

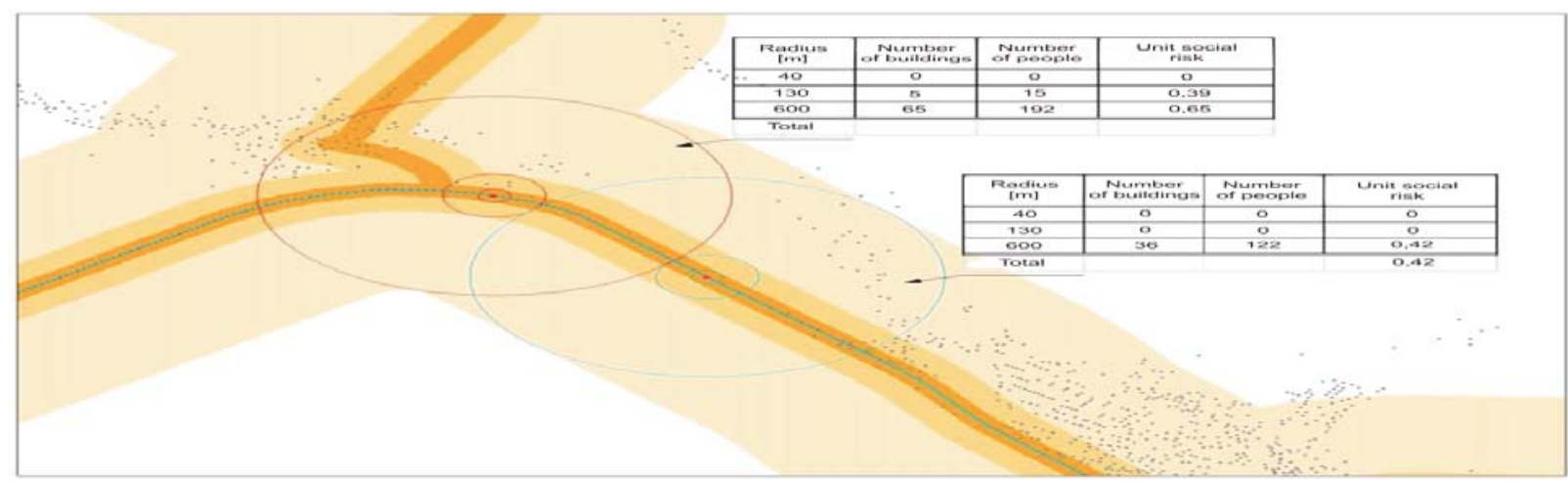

Fig. 5 Individual risk distribution nearby the traffic route

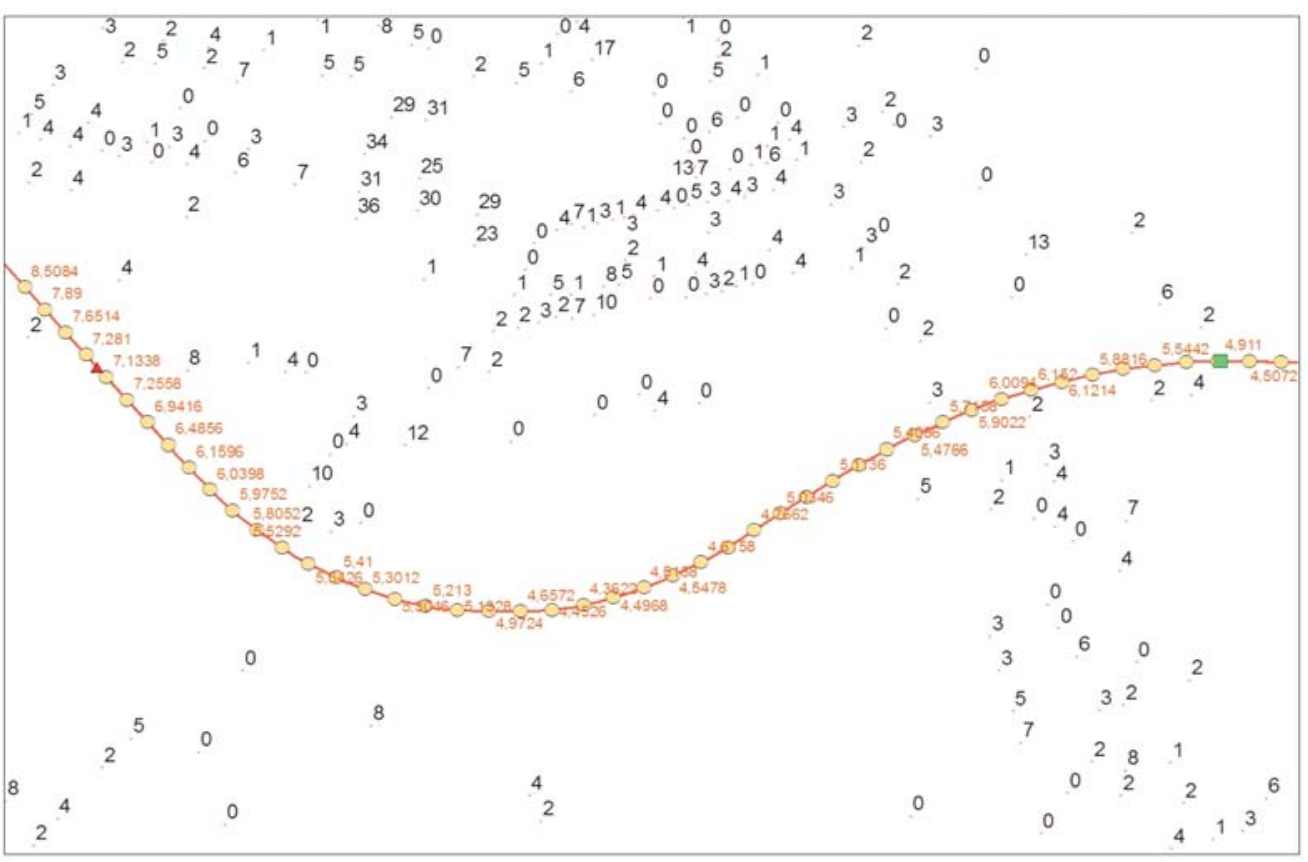

Fig. 6 Address points, number of people, unit social risk for single road parts $(20 \mathrm{~m})$ 


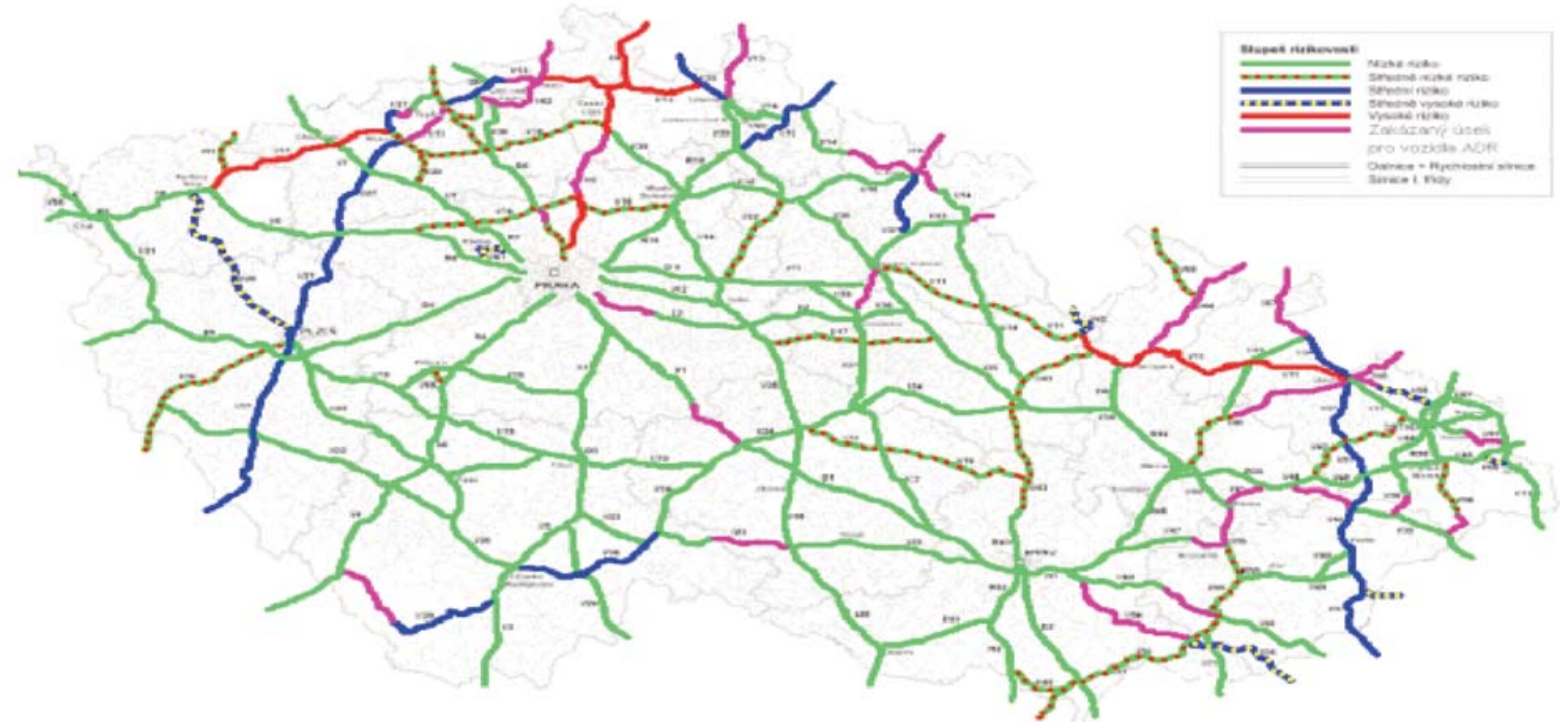

Fig. 7 Possible version of selected road network segments for dangerous goods transport with implemented exposures in the Czech Republic (pink colour - dangerous goods transport ban according to law)

$P_{\varphi}(r)_{i}$ average death (injury) probability/(lethal dose) in a cloud axis,

$i \quad$ route segment index.

Individual risk can be determined in point network according to the death or injury impact contours, which surround the traffic route. The illustration of network distribution and final individual risk maps can be found in the next figure below. It is evident that the enhanced risk is the curve inside the meandering road where the interest point can be affected by accidents in more parts of the traffic route.

\subsection{Road network assessment for the transportation of dangerous goods}

According to the risk maps, the road segments can be determined [6], [7]. Based on those road segments is it possible to define a route with minimal social and individual risks. It is possible to:

1. Displays risk maps

2. Uses the data/maps for assessment in the transportation of dangerous goods and to find recommended routes.

\section{Conclusion}

This paper represents the present status of the project BIOTRA (2B08011). Currently half of the research was conducted therefore partial outcomes have been presented. Soon it will be possible to present complex software tools, which would enable users to compile route maps for the transport of dangerous goods as well as to simulating the risks connected to transport and to determine individual and social risks.

It is important to note that the simulation model will include solutions for all parts and at the same time support cooperation between its parts with minimal demand for "manual" operation with the data. According to evaluated consequence (toxicity, fire, and explosion) is it possible to precede to the individual risk calculations. Toxicity defines human death or injury probability presented via cloud axis and effective cloud width. The fire effects depend on cloud dimensions within combustion limits, heat flux and thermal exposure dosage. The system will be designed with the focus placed on the shock front calculations for after a possible explosion.

The software tool enables the user to get the data in a form which can be used for probability averaging through the use of frequencies of particular meteorological situation. This system can also be used for the creation of individual risk maps. The output possibilities are designed to correspond with different demands for following graphic and tabular proceedings.

The paper was supported by project MŠMT 2B08011 BIOTRA.

\section{References}

[1] BUBNIK, J., KEDER, J., MACOUN, J., MANAK. J.: System of Modelling Stationary Sources (in Czech), Prague, 1998. 
[2] CROWL, D. A., LOUVAR, J. F. Chemical Process Safety: Fundamentals with Application, PTR Prentice - Hall, Inc. A. Simon \& Schuster Company, Englewood Cliffts, New Jersey, 1990.

[3] CIZLAK, M., DVORAK, Z.: Specification of Extraordinary Events During Transportation of Dangerous Goods in Slovakia (in Slovak). In: Solving Crisis Situations in Specific Condition, 2007, Zilina. ISBN 978-80-8070-700-2. p. 97-100.

[4] DVORAK, Z.: New Aspects of Transporting Dangerous Material and Oversized Shipments, In: Transport 2006, Sofia, 2006, p. I-39-42, ISBN 954-12-0130-X.

[5] DVORAK, Z., HAVLICEK, J., SOUSEK, R.: Evaluation of Public Risk Connected to Evasion of Dangerous Substances Using GIS, In: Mechanics Transport Communications, Academic Journal, Sofia, ISSN1312-3823, issue 3 (2009), p. V-1-V-3.

[6] MITCHEL, A. The ESRI Guide to GIS Analysis, In: Spatial Measurements \& Statistics, Redlands, Vol. 2, California : ESRI Press, 2005. ISBN 978-1-58948-116-9.

[7] Methods for the Calculation of Physical Effects Resulting from Releases of Hazardous Materials (liquids and gases), Yellow Book. Third Edition - second print, Committee for the Prevention of Disasters (CPR), Directorate - General of Labour of the Ministry of Social Affairs, The Hague, 2005. CPR 14E.

[8] Guidelines for Quantitative Risk Assessment, Purple book. Committee for the Prevention of Disasters (CPR), Directorate - General of Labour of the Ministry of Social Affairs, The Hague, 1999. CPR 18E.

[9] Statistical Yearbooks of Slovak Republic. 